\title{
ETIOLOGY AND PROGNOSIS OF NEONATAL CONVULSIONS
}

Researchers at Istanbul University, Turkey evaluated etiologic and risk factors affecting long-term neurologic outcome in newborns with neonatal seizures. Of 112 newborns referred to the Department of Pediatric Neurology from Jan 1, 2007-Dec 31, 2009, 33 were preterm and 79 full-term (41 female, 71 male). The rate of seizures within the first 24 hours was higher following perinatal asphyxia $(p=0.0001)$. Status epilepticus occurred in 26 patients $(23.2 \%)$. Seizures were tonic in 21 patients $(18.8 \%)$, subtle in 18 $(16.1 \%)$, myoclonic in $15(13.4 \%)$, and clonic in $15(13.4 \%)$. EEG was normal or slightly abnormal in 69 patients $(61.6 \%)$, and very abnormal in $16(14.3 \%)$. Cranial imaging detected parenchymal hemorrhage in 22 cases $(19.7 \%)$. Seizures responded to phenobarbital in 73 patients $(65.2 \%)$. Etiologic factors included perinatal asphyxia in 22 cases $(28.6 \%)$, intracranial bleeding in $19(17 \%)$, metabolic disease in $12(10.7 \%)$, unknown in $10(8.9 \%)$, and miscellaneous. Prognosis was poorer in patients with perinatal asphyxia compared to those with metabolic disorders or unknown etiology. Epilepsy and global developmental delay, low Bayley cognitive and motor scores, and low language scores were more frequent following perinatal asphyxia compared to the group with metabolic disease or seizures of unknown etiology. Epilepsy developed in $35.7 \%$ patients, cerebral palsy in $27.6 \%$, and global developmental delay in $50.8 \%$. Prognostic factors in neurologic outcome included etiology, Apgar score, resuscitation at birth, abnormal EEG, neonatal status epilepticus, duration of AEDs, and response to acute treatment. (Yildiz EP, That B, Ekici B, et al. Evaluation of etiologic and prognostic factors in neonatal convulsions. Pediatr Neurol 2012 Sep;47(3):186-92). (Respond: Dr Ekici. E-mail: ekicibaris@yahoo.com).

COMMENT. Perinatal asphyxia is the most common cause of neonatal seizures and a risk factor for epilepsy, cerebral palsy and developmental delay. In one previous study of prognostic factors in 120 term infants with neonatal seizures, the prevalence of epilepsy, cerebral palsy and global developmental delay was $32 \%, 31 \%$, and $43 \%$, respectively. (Garfinkle J, Shevell MI. Pediatr Neurol 2011 Feb;44(2):88-96). It is unclear whether the cognitive impairment and developmental delay are in part the result of seizures or AED side effects. A small but significant depressant effect of long-term phenobarbital on cognitive function of children with febrile seizures is well known (Farwell JR et al. N Engl J Med 1990 Feb 8;322(6):364-9). Duration of AEDs was a prognostic risk factor in outcome in the present study. Further controlled studies of the long-term effects of AEDs on patients with neonatal seizures and epilepsy are required.

Status epilepticus and EEG abnormalities in the first year of life predict worse developmental outcome in children with Dravet syndrome also, complications that may be amenable to more specific therapy. (Brunklaus A et al. Brain 2012 Aug;135(Pt 8):2329-36). Dravet syndrome is a form of epilepsy associated with cognitive and motor disorders, resulting in encephalopathy. (Dravet C. Brain 2012 Aug;135(Pt 8):2309-11). 